\title{
LA ENSEÑANZA Y APRENDIZAJE DEL INGLÉS COMO LENGUA AÑADIDA: ESTUDIO DE CASO DE LA COMUNIDAD INMIGRANTE EN BRADFORD
}

Sonia Peña', José Luis Ortega²

\section{RESUMEN}

Hasta la fecha, numerosas investigaciones se han llevado a cabo sobre la enseñanza del inglés como lengua añadida (EAL en inglés). Sin embargo, parecen no haber sido suficientes para cubrir las necesidades que este tipo de alumnado y profesorado requiere. Este estudio trata de analizar lo que está sucediendo actualmente en las aulas. Se llevó a cabo en varios colegios de secundaria en Bradford (GB), donde más de la mitad del centro son alumnos EAL, con una participación de 117 sujetos. El principal objetivo es el de analizar qué métodos se utilizan en la enseñanza del inglés como lengua añadida y realizar una propuesta de mejora.

Palabras clave: inmigración, integración, EAL, minorías étnicas, diversidad.

\section{ABSTRAC}

To date, several investigations have been carried out about teaching English as an additional language (EAL). However, they have not been enough to cover the needs that this type of students and teachers require. This study attempts to analyze what is currently happening in the classroom. This study took place in several secondary schools in Bradford (UK), where more than half of the population are EAL students and a total of 117 people participated in the study. The main objective is to analyze what methods are used in teaching EAL and suggest an improvement proposal.

Key words: immigration, integration, $E A L$, ethnic minority groups, diversity.

\footnotetext{
${ }^{1}$ Universidad de Granada, Facultad Ciencias de la Educación, Granada, España (soniapg85@gmail.com). 2 Universidad de Granada, Facultad Ciencias de la Educación, Granada, España (joseluisortegamartin@gmail.com).
} 


\section{INTRODUCCIÓN}

... Si tú no emigraste, emigró tu padre, y si tu padre no necesitó mudar de sitio fue tu abuelo, antes, no tuvo otro remedio que ir, cargando la vida sobre la espalda, en busca de la comida que su propia tierra le negaba.

\section{José Saramago}

Según los datos publicados por la Oficina Nacional de Estadística (ONS) ${ }^{3}$ en el (Annual Mid-year Population Estimates, 2013), Inglaterra es un país que ha registrado el fenómeno de la inmigración durante siglos, pero ha sido en las últimas décadas donde ha ejercido como puerta principal de entrada para la inmigración procedente de Europa y Asia principalmente. En la actualidad, estos movimientos migratorios se ven reflejados en la población escolar en una diversidad social y cultural creciente. Estos cambios significativos, han motivado que durante los últimos años, en el sistema educativo hayan surgido nuevas necesidades a las que atender y respuestas que ofrecer a dichas circunstancias. En este sentido, el centro educativo es el lugar que debemos usar para llevar a cabo una integración entre las diversas procedencias y culturas, que permita cruzar lazos de confianza, respeto e igualdad por encima de las diferencias. Para el gobierno británico, apoyar a los estudiantes que tienen inglés como lengua añadida es un área de trabajo de la máxima prioridad (EAL Nexus Website, British (ouncil ${ }^{4}$ ). Su objetivo central es ayudar a que los estudiantes inmigrantes puedan acceder y aprender positivamente en las escuelas, en la comunidad y en la sociedad, promoviendo así el diálogo intercultural y la cohesión social.

\section{MARCO TEÓRICO}

En Inglaterra, es habitual encontrar aulas de primaria y de secundaria donde son muy pocos los alumnos que tienen el inglés como primera lengua en casa. Por las cifras tan altas que han alcanzado en los últimos años, el gobierno se ha visto en la necesidad de apoyar y atender a aquellos alumnos que se encuentran en esa situación. La gestión en las aulas de inclusión en los colegios públi-

\footnotetext{
${ }^{3}$ La Oficina Nacional de Estadísticas (ONS) es una autoridad británica que se encarga de la creación y publicación de estadísticas en los cambios de la economía, población y sociedad a diferentes niveles nacionales y locales.

${ }^{4}$ El Consejo Británico (en inglés British Council ) es un instituto cultural público equivalente al Instituto Cervantes, cuya misión es difundir el conocimiento de la lengua inglesa y su cultura mediante la formación y otras actividades educativas. La EAL Nexus website, es una plataforma que da apoyo exclusivamente al profesorado y alumnos EAL, con una gran variedad de recursos y materiales a disponibilidad pública.
} 
cos tanto de primaria como de secundaria, se lleva a cabo por medio del English as an Additional Language Programme (EAL), que tiene como objetivo lograr que este alumnado, los new-to-English students, pueda seguir de manera normal el National Curriculum en el menor tiempo posible. En el apartado de inglés como lengua añadida en Inglaterra, analizaremos en detalle el programa EAL. Hasta la fecha autores como Stephen Talmy (2011), Angela Creese (2005) y Jean Conteh (2012), han llevado a cabo investigaciones sobre la enseñanza y aprendizaje del inglés como lengua añadida. Muchas de sus investigaciones son guías dirigidas al profesorado que hablan de diferentes estrategias de enseñanza (Conteh, 2015), de la formación necesaria del profesorado (Conteh, 2012), de criterios de evaluación (Creese, 2012) y de las necesidades del alumnado EAL (Leung y Creese, 2010). Además existen dos importantes entidades en Reino Unido que ofrecen apoyo escolar, formación, opciones de investigación y una variedad de recursos "online": Naldic (National Association for Language Development in the Curriculum) y EAL Nexus Website de la British Council.

No obstante, parece que estos recursos no son suficientes para cubrir todas la necesidades que este tipo de profesorado y alumnado requiere. Con este estudio, intentamos analizar lo que sucede actualmente en las aulas EAL, e intenta dar respuestas a cuestiones aún sin resolver como podrían ser las siguientes: ¿Hasta qué punto el programa EAL implementado actualmente en Inglaterra puede integrar al alumnado inmigrante en el currículo nacional de manera eficaz y eficiente? Si es así, ¿cuáles son las claves de su éxito? y si no, ¿Cuáles son algunas de las áreas que se podrían mejorar?

Con el presente estudio, proponemos analizar qué métodos se usan para la enseñanza del inglés como lengua añadida, estudiar en profundidad el programa de adaptación, el nivel de importancia que se da a las raíces culturales y realizar una propuesta de mejora para que el alumnado inmigrante se sienta parte de la nueva cultura, respetando y manteniendo la de origen. La investigación fue llevada a cabo en las aulas EAL en varios colegios de secundaria en Bradford, donde más de la mitad del centro son alumnos EAL. El diseño del estudio es mixto, cualitativo y cuantitativo, no experimental, basado en el estudio de caso descriptivo como metodología de investigación. 


\section{LA INMIGRACIÓN EN EL REINO UNIDO}

El Reino Unido ha sido un punto de llegada de inmigrantes durante siglos y, especialmente, en las últimas décadas. Durante los años 1960 y 1970 hubo más personas que emigraban desde el Reino Unido a diferencia de los que llegaban para vivir en él. Según los datos publicados el 26 de junio de 2014 por la ONS en el Annual Mid-year Population Estimates 2013, refleja que la migración neta se mantuvo en un nivel relativamente bajo durante la década de 1980 y principios de 1990.

Desde 1994, la inmigración al Reino Unido se mantuvo estable, pero después de 1997 creció bruscamente. Durante la primera década del siglo XXI, la inmigración neta tuvo un aumento significativo de 150.000 a 300.000 personas aproximadamente. Este crecimiento se produjo por la incorporación de diez nuevos países miembros a la Unión Europea el 1 de mayo de 2004: República Checa, Chipre, Eslovaquia, Eslovenia, Estonia, Hungría, Letonia, Lituania, Malta y Polonia.

La Oficina Nacional de Estadísticas (ONS) en colaboración con el Ministerio del Interior (Home Office en inglés) y el Departamento de Trabajo y Pensiones (en inglés Department for Work and Pensions ) publica el informe Migration Statistic Quartely Report (MSQR) con las estimaciones anuales de la migración internacional a largo plazo en el Reino Unido.

La ONS define a "long- term International Migration" como:

A person who moves to a country other than that of his or her usual residence for a period of at least a year (12 months), so that country of destination effectively becomes his or her new country of usual residence.

\section{Inmigración por áreas}

En el Reino Unido, la inmigración internacional a largo plazo puede producirse por diferentes motivos: inmigración por estudios, trabajo, familias, amigos o refugio. La tercera o cuarta parte del total de la inmigración en Gran Bretaña, es producida por motivos de trabajo o estudios principalmente, según afirman los datos facilitados por la MSQR en febrero de 2015.

El último informe de Migration Statistic Quartely Report (MSQR) publicado el 26 de febrero de 2015, refleja un crecimiento estadísticamente significativo de 624.000 personas inmigradas al Reino Unido respecto a las 530.000 en el año anterior (2014), cifras recogidas hasta septiembre con el cierre de cada año. Como se ha mencionado previamente este crecimiento fue producido por la in- 
corporación de nuevos países miembros a la Unión Europea. Como detalla International Passenger Survey (IPS) en el último informe de MSQR febrero de 2015, se estima que el $51 \%$, 30\% y el $16 \%$ del total de los inmigrantes Europeos en el Reino Unido pertenecen a los primeros 15 países de la Unión Europea (EU15), a los 8 países de Europa de Este que se incorporaron en 2004 (EU8) y a Bulgaria y Rumania que se unieron en 2007 (EU2).

En relación con los inmigrantes no europeos, también se produjo, hasta septiembre 2013/2014 un crecimiento respecto al año anterior de 243.000 a 292.000. De los Estados que forman el Reino Unido, Inglaterra es el que acoge el mayor número de inmigrantes llegados al país. El Migration Statistic Quartely Report del 27 de noviembre de 2014, afirmaba que 462.000 inmigrantes llegaron a Inglaterra en el 2013, lo que representa un $88 \%$ del total de la población extranjera (530.000) en Gran Bretaña hasta junio de 2014 con el cierre del año. Las proporciones respecto a los inmigrantes que llegaron a Gales, Escocia e Irlanda del Norte eran del 4\%, $6 \%$ y $2 \%$ respectivamente. Dentro de Inglaterra, 140.000 personas inmigraron a Londres, cifra que no representa un crecimiento estadísticamente significativo en comparación con 2012, (MSQR noviembre 2014). La International Passenger Survey indica que Londres es la puerta de entrada internacional al Reino Unido y, por tanto, es un punto de transición previo al fijar una residencia en cualquier otra parte del Reino Unido, (Long-Term International Migration Methodology Document, febrero de 2015). El Sureste de Inglaterra (a las afueras de Londres) recibió el mayor número de inmigrantes, 62.000 en el 2013. En total, Londres y el Sureste recibieron el $38 \%$ del total de los inmigrantes llegados al Reino Unido en 2013, cifra similar a años anteriores. Las regiones del Este y Nordeste recibieron el mayor número de inmigrantes a las afueras de Londres y del sureste del país, con 56.000 y 51.000 respectivamente. En 2013, Inglaterra también obtuvo el mayor número de emigrantes, 272.000 personas emigraron de Inglaterra a países fuera del Reino Unido, lo que representa el $86 \%$ de todos los emigrantes procedentes de UK. Gales, Escocia y el Norte de Irlanda representan el 3\%, 8\% y $3 \%$, (MSQR, noviembre 2014). En Inglaterra, la gran diversidad de minorías étnicas es considerable. Leicester acoge el mayor número de residentes nacidos en India; Ealing en el sur de Londres tiene la cifra más alta de inmigrantes polacos; Slough y Bradford tienen el número más alto de residentes nacidos en Pakistán. Existe una gran cantidad de residentes con pasaportes europeos en Boston Lincolnshire, Peterborough y en otros muchos distritos de Londres. 


\section{Lenguas de Inglaterra}

Es evidente que el uso de otras lenguas distintas al inglés es una parte del patrimonio cultural de un porcentaje importante y creciente de la población británica. El Centre of Dynamics of Ethnicity (CODE) de la Universidad de Manchester, publicó un informe con los datos recopilados en el censo de 2011 sobre las principales lenguas de Inglaterra. 138.000 residentes entre Inglaterra y el País de Gales declararon no hablar inglés, la lengua principal más representada fue con diferencia el polaco con 546.000 hablantes, seguida por el panjabi $6,6 \%$ y el urdu 6,5\% del total de los ciudadanos que representaron hablar otra lengua distinta al inglés.

De los grupos más numerosos de lenguas diferentes al inglés se encuentran su mayoría dentro de Londres, con un 22\% o 1.7 millones de residentes. Fuera de la capital, los porcentajes más altos se encuentran en áreas como Leicester (28\%), Manchester (17\%), Peterborough (16\%), Birmingham (15\%) y Bradford (15\%). Según el CODE las principales 20 lenguas habladas en Inglaterra y Gales por número de hablantes en 2011, fueron:

$\begin{array}{ll}\text { Polaco: } & 546.174 \\ \text { Urdu: } & 268.680 \\ \text { Panyabi: } & 273.231 \\ \text { Bengali: } & 221.403\end{array}$

$\begin{array}{lr}\text { Árabe: } & 231.094 \\ \text { Francés: } & 159.290 \\ \text { Chino: } & 147.099 \\ \text { Portugués: } & 141.052 \\ \text { Español: } & 133.453 \\ \text { Tamil: } & 120.222 \\ \text { Turco: } & 100.689 \\ \text { Italiano: } & 99.423\end{array}$

Más del 50\% de los siguientes distritos, no representaron el inglés como lengua principal en el Censo 2011, (CODE): Latimern, Spinney Hills y Belgrave en Leicester; Southall Broadway y Southall verde en Ealing; Green Street Oriente y Wall End en Newham; Alperton y Wembley en el centro de Brent; Peterborough central; Green Street West y East Ham Norte.

Estas áreas se ubican en algunas de las zonas con mayor diversidad étnica de Inglaterra y Gales. La mayoría de ellos acogen grandes grupos de personas del sur de Asia: en Latimer (Leicester), el 74\% de los residentes declararon el Hindi como lengua principal en el Censo de 2011. Las principales lenguas agrupadas a nivel de distrito con más de 600 hablantes fueron:

- Yiddish, el 75\% se declaró en los barrios londinenses de Hackney, $12 \%$ en Salford y $10 \%$ en Haringey.

- Pahari / Mirpuri / Potwari : el 50\% de todos los que la hablan viven en Birmingham, el 6,4\% en Roch- 
dale y 4,8\% en Bradford.

- Hebreo: el 25\% vive en Barnet, en Hackney y Camden, un 10\% en Westminster y Haringey 4,3\%.

- La categoría que combina todas las lenguas de Australia y Oceanía se encuentran en Wiltshire 17\% y en Richmondshire 6\%.

Otros grandes grupos de lenguas incluyen: krio, turco, gujarati y bengalí. Las lenguas menos agrupadas, es decir, lenguas cuyos hablantes son los que más dispersos están en los distritos de Inglaterra y Gales son tailandés, holandés y polaco, junto con la lengua de señas británicas (Sign Language en inglés), CoDE 2013.

\section{Un país multicultural}

La rica diversidad de culturas, sociedad y lenguas que se ha desarrollado durante siglos en Inglaterra se ve reflejada en las escuelas. Hay más de un millón de niños entre los 5 y 18 años de edad que hablan más de 360 lenguas entre ellos. Más del 25 por ciento de los alumnos pertenece a una minoría étnica y casi uno de cada seis alumnos habla inglés como lengua añadida. Cada mes de enero, se realiza el Censo escolar en Inglaterra, de donde se desprenden datos fundamentales para realizar los cálculos de financiación escolar, que incluyen: Dedicated Schools Grand Funding; Funding Academies; Pupil Premium Funding y Capital Funding Allocations. Los datos aportados por el último Censo escolar se detallan en el informe publicado por el Departamento de Educación de Reino Unido, Schools, pupils and their characteristic: January 2014 y refleja lo siguiente:

En las escuelas de secundaria financiadas por el Estado (state-funded schools en inglés) un 14,3\% de los alumnos representaron otra lengua distinta al inglés, lo que muestra un aumento respecto a enero de 2013 (13,6\%).

Más de la mitad de las escuelas financiadas por el Estado se convirtieron en Academy $^{5}$, en enero de 2014, representaban un $57 \%$, lo que muestra un pequeño crecimiento de menos del 50 por ciento respecto a 2013. 29,5\% de los alumnos en las escuelas de primaria financiadas por el estado, fueron clasificados en un grupo étnico, lo que muestra un aumento respecto a enero de 2013 (28,5\%). Los alumnos pertenecientes a minorías étnicas forman el 60\% del crecimiento total de las escuelas de primaria.

\footnotetext{
${ }^{5}$ Las academies son escuelas independientes, que reciben la financiación directa del gobierno en lugar de las autoridades locales. El funcionamiento de la escuela recae en el director/a, son supervisadas por organismos individuales denominados "Academy trust" que le ofrecen apoyo y sugerencias. Las academies tienen más libertad que otras escuelas estatales sobre su financiación y planes de estudios. El profesorado no necesita seguir las condiciones nacionales de pago.
} 
Los grupos étnicos con el mayor crecimiento hasta enero de 2014 fueron: White British subió a 43.000, other White background 21.000, Mixed 13.600, Asian 13.300 y Chinese 9.000. En las escuelas de secundarias financiadas por el Estado, $25,3 \%$ de los alumnos fueron clasificados en un grupo étnico, con un aumento respecto a enero de 2013 (24,2\%). Desde el 2009, un poco menos del $20 \%$ de los alumnos de state-funded secondary schools eran de orígenes étnicos minoritarios, por lo que en 5 años ha tenido un crecimiento de más del 25\%. Según los datos publicado por Naldic (EAL statistic), los alumnos que tienen el inglés como lengua añadida, conocidos como EAL learners en las escuelas de primaria y secundaria financiadas por el Estado ha tenido un incremento anual y constante que se ha producido desde 1997 hasta la fecha. En 1997 se calculó 257.000 alumnos en las escuelas de primaria y 225.000 en las escuelas de secundaria, hoy día llegan a 665.000 alumnos de primaria y 460.000 de secundaria. Desde enero de 2013 a 2014 se produjo un aumento de $0,6 \%$ en las escuelas de primaria y $0,7 \%$ en las escuelas de secundaria. El número y el porcentaje de alumnos bilingües o EAL, varía significativamente por todo el país según el tipo de escuela.
En las escuelas rurales son muy pocos los alumnos EAL escolarizados, la gran mayoría se encuentra en las escuelas urbanas llegando casi al 100\% (EAL Nexus, British Council).

En los datos recogidos por el informe Schools, pupis and their characteristic de 2014, las 5 localidades con el mayor número de alumnos EAL de primaria y secundaria fueron:

\section{Escuelas financiadas por el Estado}

\section{Primaria}

Birmingham: $\quad 37.605$

Newham: $\quad 20.122$

Ealing: $\quad 15.207$

Tower Hamlets: 14.458

Bradbord: $\quad 13.788$

\section{Secundaria}

Birmingham: 25.589

Newham: $\quad 13.127$

Redbridge: 13.079 Bradford: $\quad 12.846$ Brent: $\quad 10.350$
Además, el menor número de alumnos EAL en las escuelas de primaria y secundaria de Inglaterra se encuentran en Redcar y Cleveland con 93 alumnos en primaria y 118 en secundaria; Halton con 114 en primaria y 75 en secundaria y Rutland con 72 en primaria y 50 en secundaria. El condado de Rutland acoge el menor número de alumnos EAL de toda Inglaterra.

\section{EL INGLÉS COMO LENGUA AÑADIDA EN INGLATERRA}

Como hemos mencionado al principio del estudio, la enseñanza del inglés como lengua añadida conocida en inglés como English as an Additional Language program es impartida en los colegios de Primaria y Secundaria finan- 
ciados por el Estado. Este programa tiene como objetivo lograr que alumnado EAL pueda incorporarse al plan nacional de estudios con total normalidad y en igualdad de condiciones al alumnado local.

El Ministerio de Educación, (en inglés Department of Education (DFE)) define la primera lengua como:

as the language to which a child was initially exposed during early development and continues to be exposed in the home or in the community.

Para la gran mayoría de los alumnos EAL esto significa que si comienzan la escuela a los 3-5 años de edad como alumnos EAL, serán estudiantes de EAL a lo largo de toda su etapa escolar.

No existe ningún plan de estudios acordado a nivel nacional para los alumnos EAL. Desde mediados de 1980, la ley inglesa señalaba que los aprendices EAL junto con los demás alumnos deben tener el mismo acceso al plan de estudios nacional sin ningún currículo específico. En Inglaterra, las segundas lenguas son reconocidas con éxito, sin embargo la lengua predilecta en las escuelas y para todo alumnado es el inglés, a través de él se alcanzará el logro académico. La política de alumnos que estudia a los EAL en el aula ordinaria a través del Plan Na- cional de Estudios, plantea cuestiones no solo de lengua y de pedagogía, sino también de los derechos y beneficios de la integración social y la igualdad de acceso al sistema educativo (EAL Nexus, British (ouncil).

A través del Plan Nacional de Estudios, el DFE espera una enseñanza eficaz para los alumnos EAL, que se lleve a cabo dentro del plan de estudios y que el profesorado tenga presente lo siguiente:

1. Las necesidades de los alumnos cuya lengua materna no es el inglés. En este seguimiento, se considerará la edad del alumno, el tiempo que lleva en el país, la experiencia educativa previa y la habilidad en otros idiomas.

2. Planificar las clases de tal modo que puedan ayudar a los alumnos a desarrollar su inglés y dar el apoyo que necesiten para participar en las demás materias.

En septiembre de 2013, el DFE planteó para el nuevo curso escolar 2014/2015 no ofrecer apoyo específico para los alumnos EAL, sino que propusieron desarrollar lo siguiente:

1. Tener altas expectativas de todos los estudiantes, independientemente de sus antecedentes o necesidades.

2. Dar plena libertad a las escuelas 
para implementar las medidas necesarias.

3. Tener un nivel alto de responsabilidades a través de la Office for Standards in Education, Children's Services and Skills (Ofsted).

\section{Cómo evaluar a los alumnos de inglés como lengua añadida}

No existe un sistema nacional de evaluación para medir el desarrollo y aprendizaje de los alumnos EAL. La guía más reciente publicada por Ofsted ${ }^{6}$ declaró que la escuela debe supervisar el logro y el progreso de los alumnos que pueden estar en la primera etapa del aprendizaje del inglés. Para ello, se sugiere utilizar los descriptores de nivel que incluye el Currículo Nacional 2000 (en inglés A language in common: assessing English as an additional language,). Aunque dicha escala no está oficialmente legalizada, fue plenamente recomendada por el Department for children, schools and families (dcfs). Además, la escuela también deberá tomar medidas para evaluar las competencias y la alfabetización de los alumnos en su lengua materna y haber comprobado los conocimientos previos y la experiencia en otras materias.

\section{Fondos para el alumnado inmigrante}

Desde abril de 2013, un factor EAL podría incluirse en las fórmulas de financiación de las escuelas inglesas para satisfacer las necesidades de los alumnos bilingües. Este factor se limita a los alumnos bilingües que han estado escolarizados por un período máximo de tres años. The local schools Forum puede decidir si debe o no incluir un factor EAL en su fórmula, si este factor incluye a los alumnos bilingües que han estado escolarizados por uno, dos o tres años y el valor en efectivo de este factor para los alumnos de primaria y de secundaria.

Las autoridades locales no están obligadas a incluir este factor, ni tampoco existe un mecanismo que delegue sobre el uso de la financiación EAL en las escuelas. Estas no están obligadas a utilizar estos fondos para satisfacer las necesidades de los alumnos bilingües, lo que contrasta con la exigencia de dar cuenta sobre el uso de sus fondos y sus efectos. Para el curso escolar 2014/15, el $87 \%$ de todas las autoridades locales optaron por incluir el factor EAL en su fórmula de financiación. Un 23\% de las autoridades locales optaron por incluir a los alumnos que habían estado escolarizados por solo uno o dos años, en lugar de los tres años completos. El valor que

\footnotetext{
${ }^{6}$ Ofsted, se encarga de realizar inspecciones regulares de cada escuela en Inglaterra, lo que deriva a desarrollar un informe de la eficacia del centro y será libremente publicado. Un informe desfavorable puede incluir una recomendación para mejorar el funcionamiento del centro y cómo poder llegar al éxito. El objetivo principal es lograr la excelencia en la educación para todos los estudiantes en general.
} 
se asigna al factor EAL es de 250 hasta 1.000 libras por alumno. Por lo general, todas las autoridades locales están asignando menos del $5 \%$ de financiación a este factor, con un promedio de 0,9\%. En el curso escolar 2014/15, un total de 243 millones de libras se delegó a las escuelas a través del factor EAL,(Naldic, EAL Funding). Para el siguiente curso escolar 2015/16, el gobierno ha establecido un nivel mínimo que será de 466 libras para Primaria y 1.130 libras para Secundaria. Este nivel mínimo de financiación se destinará a los alumnos que entraron en el sistema escolar en los últimos tres años.

\section{Los alumnos de inglés como lengua añadida en Bradford}

Bradford es el cuarto distrito metropolitano más grande en términos de población en Inglaterra, después de Birmingham, Sheffield y Leeds. En los dos últimos años, la población ha crecido un $0,6 \%$, lo que es más lento que el promedio regional de $0,9 \%$ y la media nacional de 1,4\%. El crecimiento de la población en Bradford es inferior a otras ciudades como Birminghan, Manchester o Londres, (Bradford Metropolitan District Counci).La población de Bradford es totalmente multicultural, un 63,9\% de la población se identifica como White British.

Bradford cuenta con la mayor cifra de origen étnico paquistaní de Inglate- rra con un 20,3\%. Uno de los grupos étnicos más reciente llegados a Bradford pertenece a Europa Central y Oriental, concretamente de Eslovaquia, República Checa y Polonia. Por lo general, son familias de origen de etnia gitana, de Eslovaquia y República Checa. Los gitanos polacos, rumanos y húngaros son menores en número. El estado social de Bradford es de inmigrantes con motivos económicos (en inglés Economic Migrants), pero se estima que gran parte de ellos se van a afincar allí y a formar parte de la comunidad, (Bradford Metropolitan District Council).

Bradford a su vez, cuenta con familias de refugiados que se han trasladado a la zona buscando un asilo, seguridad y mejor calidad de vida, debido a una persecución por motivos de raza, religión, nacionalidad y opinión política en su país de origen, (Bradford Metropolitan District Council). Según el Censo Escolar de 2014, la ciudad de Bradford cuenta con 36.485 alumnos escolarizados en las escuelas de secundaria financiadas por el Estado, de los cuales 19.230 pertenecen a minorías étnicas. Esta cifra está formada por los alumnos que pertenecen a uno de los cinco principales grupos étnicos según las categorías del Censo: white, mixed, asian, black, chinese y any other ethnic group. Como muestran los datos recogidos en el Censo, el grupo que acoge el mayor número de alumnos respecto a las otras minorías étnicas 
es el asiático con 15.665 alumnos. De esta cifra cabe destacar los 12.911 que pertenecen al grupo pakistaní. Estos datos ratifican lo que hemos mencionado anteriormente, que el distrito de Bradford recibe el mayor número de ciudadanos pakistaníes. Además, solo 349 alumnos corresponden al grupo Gypsy/
Roma (gitanos) lo que certifica que muchas familias gitanas no declaran su grupo étnico en las escuelas ni en otras entidades públicas por miedo a la discriminación, National Association of Teachers of Travellers + Other Professionals (Natt+).

\section{METODOLOGÍA}

\section{Muestra}

La muestra ha sido seleccionada según un tipo de muestreo, no probabilístico de sujetos voluntarios, puesto que la elección de ellos dependió del criterio de posibilidad de acceder al alumnado y al profesorado. Un total de 117 participantes han colaborado, lo que corresponde a 101 alumnos y 16 profesores. La recogida de datos se llevó a cabo durante la primavera de 2014 en 4 colegios públicos de secundaria en Bradford (Inglaterra). La elección de dichos centros se tomó tras analizar el total de alumnos EAL escolarizados y seleccionar los centros con el mayor número.

\section{Instrumentos}

Para la toma de datos, se aplicaron observaciones participativas en el aula, las personas encargadas de pasar los cuestionarios se identificaron de antemano y participaban en la clase, a la vez que realizaban las observaciones e inter- pretaciones. También se elaboraron dos cuestionaros cerrados, tanto para el profesorado como para el alumnado, con el fin de analizar qué materiales y recursos utilizan los docentes en las aulas EAL. En la primavera de 2013, se realizó la prueba piloto de la encuesta para consolidar y probar su funcionamiento en el campo. Dichas encuestas fueron modificadas, debido a que las preguntas no eran adecuadas para una buena comprensión de los encuestados. El cuestionario usado, fue validado siguiendo el método de triangulación de expertos.

\section{Procedimiento}

Individualmente, cada participante completó la encuesta en el mismo aula de inclusión. A aquellos alumnos que ya estaban integrados en el aula ordinaria, se les pidió que fueran a la misma. A los alumnos que necesitaron apoyo para la comprensión, se les ofreció ayuda con profesores asistentes que les traducían a su lengua materna 
para obtener resultados más precisos. El profesorado optó por realizar la encuesta fuera del centro para dedicarle el tiempo requerido.

\section{Variables empleadas}

Hemos analizado qué nivel de la cultura de origen tienen los encuestados. Para ello, hemos realizado correlaciones de las variables culturales con otras cuestiones que podían influir de manera positiva o negativa en este conocimiento. En este caso, se confeccionó una variable nueva a partir de las preguntas que tenía acerca de diferen- tes aspectos culturales y otras varias que se detallan en el cuestionario. A partir de estas, se creó una variable que mide de forma más certera el conocimiento cultural de los encuestados. Se asignó una puntuación ponderada sobre cada ítem, de forma que al final se obtenga un sumatorio ponderado de las respuestas correctas que indique un nivel de conocimiento cultural, categorizada de la siguiente forma: fail (0 a 4,99), pass (5-5.99), pass + (6-6.99), outstanding (7$7,99)$ outstanding + $(8-8,99)$. En la encuesta del profesorado se empleó el valor de la moda.

\section{ESTUDIO ESTADÍSTICO}

Se trata de una investigación mixta cualitativa y cuantitativa no experimental, basada en un estudio de caso descriptivo como método de investigación. Se ha utilizado el Alfa de Cronbach estandarizado para medir la fiabilidad de las correlaciones de los ítems, habiéndose obtenido un índice de fiabilidad de 0,742 (George y Mallery, 2003: 231).

\section{Resultados}

Respecto al funcionamiento de las aula EAL, cada centro desarrolla su propio programa de adaptación, que puede tener un período de duración, comprendido entre 2 semanas hasta 1, 2 o 3 años. Los alumnos continuarán con clases de apoyo una vez finalizado el período de adaptación, pero siempre dependerá del progreso del alumno. La mayoría de los profesores de EAL solo imparten inglés y solo un $43,8 \%$ de los encuestados han recibido formación específica para impartir en dichas aulas. Una vez más, según el centro, la elección de enseñar en las aulas EAL puede ser obligatoria $u$ opcional. Al respecto, un 50\% de los encuestados confiesa que fue opcional, frente a un $25 \%$ que afirma que fue obligatoria. El resto de participantes (25\%) optaron por no contestar.

Se estudió si los materiales más utilizados en la aulas EAL eran los mismos que los alumnos habían conside- 
rado muy apropiados o apropiados para el aprendizaje de la lengua. Tras el análisis se observó que de los materiales más utilizados, entre ellos fotocopias, pizarra digital, libros de lecturas, ordenadores y videos por lo general, los alumnos lo vaIoran como muy apropiados para el aprendizaje. Además, el material utilizado en menor frecuencia por el profesorado, en este caso el libro de texto, es valorado por el alumnado como muy apropiado. Esta opinión nos hace pensar, que al día de hoy, los alumnos optan por el método tradicional, y no asimilan que sin el uso constante del libro de texto, también se puede alcanzar el éxito y aprender la lengua.

En relación con el nivel de conocimiento de lo cultural que tienen los alumnos sobre el país en el que viven se desvela que el nivel de conocimiento acerca del país en el que residen es escaso: un $81,2 \%$ del total de los encuestados no alcanzan un conocimiento mínimo de aspectos culturales. Cabe destacar que, en un porcentaje muy alto de los casos recogidos en esta población, ninguno de los progenitores trabaja; en el $44,6 \%$ de los casos, no trabaja ni el padre ni la madre. Sobre este mismo ítem, evaluamos si el nivel cultural estaba quizás influenciado por el tiempo que llevaban viviendo en Inglaterra. Los datos obtenidos mostraron que el nivel de cultura no varía en función del tiempo, pues no influye si han vivido en Inglaterra uno o diez años según se desprende de los resultados obtenidos

\section{CONCLUSIONES GENERALES}

Tras las observaciones en las aulas, se puede constatar que el objetivo principal del programa profundización en la integración del alumnado inmigrante de manera eficazy en igualdad de condiciones al alumnado local, no se está llevando a cabo, puesto que se hace una segregación al alumnado extranjero desde su ingreso en el centro. Con ello, solo se puede conseguir que los estudiantes se sientan diferentes a los demás, ya que llevan la etiqueta de EAL students nada más llegar.
El alumnado inmigrante permanece semanas, meses, e incluso años en las aulas EAL hasta llegar a alcanzar el nivel correspondiente al currículum nacional, y con ello poder acceder a las aulas ordinarias. El hecho que los alumnos de distintas nacionalidades que comparten una misma lengua (caló) permanezcan en una misma aula, como es el caso de los alumnos eslovacos y checos estudiados, concluimos que no es la manera más adecuada para el aprendizaje de la lengua de acogida. Es fundamental que el alumnado extran- 
jero se relacione con el resto de compañeros, no solo por la adquisición lingüística, sino para la construcción de valores de buena convivencia en el ámbito escolar y social.

El apoyo al alumnado inmigrante dentro y fuera del aula ordinaria es la cuestión clave ya que hay cierto debate sobre si la separación de los estudiantes es la opción correcta o no. Llegados a este punto, cabe reflexionar sobre la manera en que reciben dicho apoyo. Como pudimos comprobar a través de las observaciones en las aulas ordinarias, el alumnado extranjero que observamos estaba ubicado al final de la clase con el profesor de apoyo sin tener relación con el resto de compañeros y ni siquiera son tenidos en cuenta para trabajos grupales.

Con el fin de fomentar la cultura y la lengua de origen, los centros ofrecen clases extraescolares en su lengua materna y organizan actividades de ocio fuera del centro. La participación de sus familias en el ámbito escolar es escasa, ya que la mayoría de ellas no han tenido buenas experiencias en el ámbito social y escolar, debido a que muchas veces se han visto discriminados a la hora de representar su nacionalidad. Estos hechos, juntos con la falta de asistencia escolar, nos llevan a pensar que los problemas que aparecen en la educación de los niños inmigrantes no son atribuidos únicamente a los propios sujetos, sino en gran parte a factores externos. Un ejemplo es la falta de integración social de sus familias o el tener que trabajar siendo menores de edad. Como dice Miller (2000), el contexto social y educativo son dos factores determinantes en el proceso de aprendizaje del alumnado inmigrante, reflejado en la autoimagen que tiene el propio extranjero de sí mismo y la figura que la escuela construye de él.

En relación con el profesorado, se debería requerir una formación especifica, puesto que la metodología que se usa en la enseñanza de inglés como lengua añadida es diferente a la empleada en la del inglés como lengua materna. En definitiva, se debería formar al profesorado en general, puesto que la mayoría de los profesores no saben adaptar sus materias para la comprensión de todo el alumnado. A continuación se plantean una serie de propuestas de mejora del programa EAL, que consideramos que debieran llevarse a cabo procurando así que la integración del alumnado inmigrante se desarrollará de manera más eficaz.

1. Recibir apoyo dentro del aula ordinaria (el permanecer en contacto con alumnos nativos aseguraría una adquisición natural de la lengua, una integración en vez de aislamiento).

2. Procurar que ningún estudiante 
sea excluido de la acción que se esté desarrollando en clase (utilizando estrategias de enseñanza inclusiva el alumno extranjero manifestará mayor interés por participar y aprender la lengua).

3. Situar al alumnado EAL con otros compañeros nativos del país de origen o que están totalmente adaptados a la lengua y la cultura potenciando así la cooperación entre todos y la implicación del grupo en su proceso de adaptación.

Con este trabajo hemos obtenido un diagnóstico de la situación actual en las aulas EAL, que ya está siendo utilizado en otras investigaciones para el diseño de intervenciones pedagógicas destinadas a mejorar la adaptación del alumnado inmigrante al currículo nacional. 


\section{BIBLIOGRAFÍA}

Constant, L. and Creese, A. (eds.)(2010). English as an additional language: Aprroaches to teaching linguistic minoriy students. Reading: University of Reading.

Conteh, J. (2012). Teaching bilingual and EAL learners in primary schools. Transforming Primary QTS. London: Sage.

Conteh, J. (2015). The EAL teaching book Promoting success for multilingual learners in primary and secondary schools. London: Sage.

Creese, A. (eds.)(2012). "Multilingual teaching and learning (online)" in NALDIC Quarterly 10.1, ISSN 1751-2190

available from www.naldic.org.uk/eal-publica tions-resources/Shop/shop-products/nq 1010, accessed 19 April

George, D. y Mallery, P. (2003). SPSS for Windows step by step: A Simple Guide and Reference. 11.0 Update (4⿳a . ed.). Boston: Allyn \& Bacon.

Miller, J. (2000). Language use, identity, and social interaction: Migrant students in Australia, Research on Language \& Social Interaction, 33, 1: 66-100.

http://www.bradford.gov.uk/bmdc/co mmunity_and_living/population, accessed 6 March, 2015.

http://www.eal.britishcouncil.org/ealsector/education-policy-eal-england, accessed 6 February, 2015. http:// www.ethnicity.ac.uk/research /outputs/briefings/dynamics-of-diversity/, accessed 10 March, 2015.

http:// www.gov.uk/government/statistics/schools-pupils-and-their-characteristics-january-2014 accessed 3 April, 2015.

http://www.migrationobservatory.ox.a c.uk/briefings/long-term-international-migration-flows-and-uk, accessed 25

March, 2015.

http://www.naldic.org.uk/researchand-information/, accessed 3 April, 2014.

http://www.naldic.org.uk/researchand-information/eal-funding, accessed 2 April, 2015.

http://www.natt.org.uk, accessed 10 April, 2015.

http://www.ons.gov.uk/ons/rel/popestimate/population-estimates-for-uk-england-and-wales-scotland-and-northern-irel and/2013/stb--mid-2013-uk-populationestimates.html, accessed 10 March, 2015. 